\title{
Novel treatments for type 2 diabetes
}

\section{THE NEED FOR NOVEL THERAPIES}

Type 2 diabetes mellitus is a major threat to health with a prevalence increasing in epidemic proportions. The World Health Organization estimates that by 2030, type 2 diabetes will affect over 360 million people worldwide. Inevitably, diabetes and its complications will strain public health resources.

Despite the availability of many antidiabetic agents, approximately $60 \%$ of patients do not achieve the target glycated hemoglobin $(\mathrm{HbA} 1 \mathrm{C})$ level of $\leq 7 \%$. Reasons for this include: noncompliance; side-effects of treatments; fear of hypoglycaemia; weight-gain; problems with dose titration of antidiabetic agents; and more stringent $\mathrm{HbA1C}$ targets set by healthcare organisations which tend to change. Clearly, additional therapeutic options are needed that will overcome these clinical shortcomings.

\section{INCRETIN THERAPEUTICS}

Incretin therapeutics, which are based on mimicking the endogenous effects of glucagon-like peptide 1 (GLP-1), have revolutionised the management of type 2 diabetes since they came into clinical use in 2006.

GLP-1 mimetics have proved popular with both physicians and patients as they offer an additional therapeutic strategy that overcomes many of the pitfalls associated with current therapies: they avoid hypoglycaemia by acting in a glucose-dependent manner; they facilitate weight loss by slowing gastric emptying and reducing satiety; and they inhibit glucagon release. GLP-1 mimetics have proved valuable in bridging the gap between patients with poor diabetic control ( $\mathrm{HbA} 1 \mathrm{c}>8 \%)$ who have failed oral hypoglycaemic therapy but are reluctant to start insulin due to the undesirable effects of weight gain and fear of hypoglycaemia. $^{1}$

The two licensed GLP-1 analogues are exenatide and liraglutide, both of which are administered subcutaneously. Exenatide is associated with a sustained 3 -year $\mathrm{HbA} 1 \mathrm{c}$ reduction of $1 \%$, and $46 \%$ of patients achieve a target $\mathrm{HbA} 1 \mathrm{c}<7 \%$. In comparative studies with metformin, sulphonylureas, and insulin glargine, exenatide has demonstrated superior HbA1c reductions. ${ }^{2}$

In comparative studies, liraglutide has demonstrated superior $\mathrm{HbA} 1 \mathrm{c}$ reductions compared to glimepiride, metformin, rosiglitazone, and insulin glargine. In a head-to-head comparative study against exenatide, liraglutide was associated with superior $\mathrm{HbA} 1 \mathrm{c}$ reductions of $0.3 \%$. $^{3}$

The popularity of GLP-1 agonists is further enhanced by clinical data, which demonstrate disease modifying effects on the adverse cardiovascular profile associated with type 2 diabetes. Exenatide is associated with $\sim 5 \mathrm{~kg}$ weight reduction; $12 \%$ reduction in serum triglycerides; $5 \%$ reduction in serum total cholesterol; $6 \%$ reduction in serum lowdensity lipoprotein cholesterol; 24\% increase in cardio-protective serum highdensity lipoprotein cholesterol; and improvement in the hepatic steatosis biomarkers (alanine aminotransferase) of $41 \% .^{4}$ Liraglutide has demonstrated similar findings in addition to achieving a $4 \%$ reduction in systolic blood pressure. ${ }^{3}$

The most common side-effects associated with exenatide include nausea, vomiting, and diarrhoea, which are dose-dependent but subside over time. Cases of acute pancreatitis have been reported but estimates indicate an incidence of $0.33-0.44$ per 1000 adults per year. ${ }^{5}$ Recently the Food and Drug Administration (FDA) issued a safety warning on exenatide associating it with the development of ischaemic renal failure. ${ }^{6}$

In general, liraglutide appears well tolerated with a lower incidence of nausea compared to exenatide. Liraglutide has been associated with thyroid hyperplasia and medullary thyroid carcinoma, which has led to a 'black-box' warning. However, this association seems confined to rodents, and to date there is no evidence of a causal relationship between liraglutide and human C-cell medullary thyroid cancer. Phase I trials of an oral agent of liraglutide are due to start this year.

Other GLP-1 analogues in clinical trial phase include longer acting compounds, which offer the advantage of once-weekly administration. These include: taspoglutide, albiglutide and lixisenatide, also known as AVE0010, and a onceweekly preparation of exenatide.

\section{AMYLIN AGONISTS}

Amylin agonists have all the incretin actions except stimulation of insulin secretion. Pramlintide is the only licensed amylin analogue and is associated with $\mathrm{HbA} 1 \mathrm{C}$ reductions of $0.5-1.0 \%$. Adverse effects are mainly nausea, which improves with continuing treatment. ${ }^{7}$

\section{DIPEPTIDYL PEPTIDASE IV INHIBITORS}

The therapeutic potential of endogenous GLP-1 is limited by its short physiologic half-life, owing to its rapid inactivation by dipeptidyl peptidase IV (DPP-IV). Several selective inhibitors of DPP-IV have been developed and those that are licensed include: sitagliptin, vildagliptin and, more recently, saxagliptin. They achieve a $0.4-0.7 \%$ HbA1c reduction over 12 months, and as monotherapy are not associated with hypoglycaemia. ${ }^{8}$ Adverse effects include nasopharyngitis and urinary tract infections. To date, 88 cases of pancreatitis associated with sitagliptin therapy have been reported to the FDA.

Other DPP-IV inhibitors, which are currently in phase III clinical trials, include linagliptin, dutogliptin, gemigliptin, and alogliptin.

\section{CENTRAL NEUROTRANSMITTER MODULATORS}

Body fat stores and insulin action are controlled by the temporal interaction of circadian neuroendocrine oscillations. Bromocriptine modulates neurotransmitter 
action in the brain. Studies using bromocriptine in type 2 diabetes have shown improvements in glycaemic control and glucose tolerance with $\mathrm{HbA1c}$ reductions of $\sim 0.56 \% .^{9}$ Despite receiving FDA approval, bromocriptine is not part of the type 2 diabetes management guidelines from the National Institute for Health and Clinical Excellence.

\section{EXPERIMENTAL AGENTS}

\section{The Glimins}

Imeglimin, an oxidative phosphorylation inhibitor, is a first in a new class of oral anti-diabetic drugs known as 'the Glimins', which target the three key defects of type 2 diabetes: insufficient insulin production; excessive hepatic gluconeogenesis; and impaired glucose uptake by skeletal muscles. Its efficacy is currently being examined in phase lla clinical trials. ${ }^{10}$

\section{Renal sodium-dependent glucose co-transporter-2 inhibitors}

The glucose reabsorption system in the kidney is mediated by renal sodiumdependent glucose co-transporter 2 (SGLT2) receptors. Most filtered glucose is reabsorbed by the low affinity, high capacity SGLT2 receptors located in the proximal renal tubule. SGLT2 inhibitors enhance urinary glucose excretion, which lowers blood glucose levels independent of insulin with $\mathrm{HbA} 1 \mathrm{c}$ reductions of $0.55-0.9 \%$; $^{11}$ examples include: remogliflozin, etabonate, sergliflozin, and dapagliflozin.

\section{Fructose 1,6-bisphosphatase inhibitors}

Excessive gluconeogenesis is central to the pathophysiology of type 2 diabetes. Recently, the use of selective fructose 1,6-bisphosphatase inhibitors, a ratecontrolling enzyme of gluconeogenesis, has been explored..$^{12}$ Current data, which illustrate glucose-lowering effects, are limited to rodent studies.

\section{Peroxisome proliferator-activated receptor $\alpha / \gamma$ ligands}

The promise for peroxisome proliferatoractivated receptor (PPAR) agonists to reduce cardiovascular risk type 2 diabetes is of continued interest. In the SYNCHRONY trial, $^{13}$ aleglitazar was associated with $\mathrm{HbA1c}$ reductions of $0.36-1.35 \%$ as well as improving adverse high-risk lipid profiles. It is now being advanced into phase III clinical studies. However, there are concerns about PPAR $\alpha / \gamma$ ligands. Two such agents, muraglitazar and tesaglitazar, were withdrawn after concerns about their association with major cardiovascular events.

\section{MBX-2982}

G-protein coupled receptor 119 (GPR119) is a receptor in the gut and pancreas that interacts with bioactive lipids to stimulate glucose-dependent incretin and insulin secretion. MBX-2982, a GPR119 agonist, which has completed three phase I clinical studies, has consistently shown clinically meaningful glucose reductions. It has now entered phase II clinical trials. ${ }^{14}$

\section{THE ROLE OF THE COMMUNITY DIABETES TEAM}

With the management of diabetes making the transition from secondary into primary care, coupled with imposing pressures of guidelines to tighten glycaemic control, GPs will soon be leading the way in optimising therapeutic strategies for patients with type 2 diabetes. The next decade is likely to be exciting with the explosive pace at which new, safer, and more effective anti-diabetic treatments are rolling off the clinical phase belt. The role of the community diabetes team in instituting these novel treatments will be essential in improving diabetes care.

\section{Jason Seewoodhary,}

Specialist Registrar in Diabetes Mellitus \& Endocrinology and General Internal Medicine, Swansea School of Medicine, University of Wales, Swansea.

\section{Stephen C Bain,}

Professor and Consultant in Diabetes Mellitus \& Endocrinology and General Internal Medicine, Swansea School of Medicine, University of Wales, Swansea.

\section{Provenance}

Commissioned, not externally peer reviewed.

\section{REFERENCES}

1. Saydah SH, Fradkin J, Cowie CC. Poor control of risk factors for vascular disease among adults with previously diagnosed diabetes. JAMA 2004; 291(3): 335-342.

2. Pratley RE, Gilbert M. Targeting incretins in type 2 diabetes: role of GLP-1 receptor agonists and DPP-4 inhibitors. Rev Diabet Stud 2008; 5(2): 73-94.

3. Hansen KB, Vilsbøll T, Knop FK. Incretin mimetics: a novel therapeutic option for patients with type 2 diabetes - a review. Diabetes Metab Syndr Obes 2010; 3: 155-163.

4. Klonoff DC, Buse JB, Nielsen LL, et al. Exenatide effects on diabetes, obesity, cardiovascular risk factors and hepatic biomarkers in patients with type 2 diabetes treated for at least 3 years. Curr Med Res Opin 2008; 24(1): 275-286.

5. Bain SC, Stephens JW. Exenatide and pancreatitis: an update. Expert Opin Drug Saf 2008; 7(6): 643-644.

6. Weise WJ, Sivanandy MS, Block CA, Comi RJ. Exenatide-associated ischemic renal failure. Diabetes Care 2009; 32(2): e22-33.

7. Schmitz O, Brock B, Rungby J. Amylin agonists: a novel approach in the treatment of diabetes. Diabetes 2004; 53 (Suppl 3): S233-S238.

8. Mikhail N. Incretin mimetics and dipeptidyl peptidase 4 inhibitors in clinical trials for the treatment of type 2 diabetes. Expert Opin Investig Drugs 2008; 17(6): 845-853.

9. Gaziano JM, Cincotta AH, O'Connor CM, et al Randomized clinical trial of quick-release bromocriptine among patients with type 2 diabetes on overall safety and cardiovascular outcomes. Diabetes Care 2010; 33(7): 1503-1508.

10. Poxel. Imeglimin. http://www.poxel.com/productpipeline/imeglimin (accessed 24 Nov 2010).

11. Chao EC, Henry RR. SGLT2 inhibition - a novel strategy for diabetes treatment. Nat Rev Drug Discov 2010; 9(7): 551-559.

12. Van Poelje PD, Potter SC, Chandramouli VC, et al. Inhibition of fructose 1,6-bisphosphatase reduces excessive endogenous glucose production and attenuates hyperglycemia in Zucker diabetic fatty rats. Diabetes 2006; 55(6): 1747-1754.

13. Henry RR, Lincoff AM, Mudaliar S, et al. Effect of the dual peroxisome proliferator-activated receptoralpha/gamma agonist aleglitazar on risk of cardiovascular disease in patients with type 2 diabetes (SYNCHRONY): a phase II, randomised, dose-ranging study. Lancet 2009; 374(9684): 126-135.

14. Metabolex. Pipeline MBX-2982. Potential oral incretin/islet therapy. Hayward, CA: Metabolex. http://www.metabolex.com/MBX-2982.html (accessed 24 Nov 2010).

DOI: 10.3399/bjgp11X548884

\section{ADDRESS FOR CORRESPONDENCE}

\section{Jason Seewoodhary}

Department of Diabetes and Endocrinology, Swansea School of Medicine, University of Wales, Swansea, The Grove Building, Swansea, SA2 8PP.

E-mail: seewoodharyj@hotmail.com 